\title{
Technology-enabled Airborne Spacing and Merging
}

\author{
Mr. James Hull, Titan Corporation, Hampton, VA \\ Dr. Bryan Barmore, NASA Langley Research Center, Hampton, VA \\ Terence Abbott, Booz Allen Hamilton, Hampton, VA
}

\section{ABSTRACT}

Over the last several decades, advances in airborne and groundside technologies have allowed the Air Traffic Service Provider (ATSP) to give safer and more efficient service, reduce workload and frequency congestion, and help accommodate a critically escalating traffic volume. These new technologies have included advanced radar displays, and data and communication automation to name a few.

In step with such advances, NASA Langley is developing a precision spacing concept designed to increase runway throughput by enabling the flight crews to manage their inter-arrival spacing from TRACON entry to the runway threshold. This concept is being developed as part of NASA's Distributed Air/Ground Traffic Management (DAG-TM) project under the Advanced Air Transportation Technologies Program.

Precision spacing is enabled by Automatic Dependent Surveillance-Broadcast (ADS-B), which provides air-to-air data exchange including position and velocity reports; real-time wind information and other necessary data. On the flight deck, a research prototype system called Airborne Merging and Spacing for Terminal Arrivals (AMSTAR) processes this information and provides speed guidance to the flight crew to achieve the desired inter-arrival spacing.

AMSTAR is designed to support current ATC operations, provide operationally acceptable system-wide increases in approach spacing performance and increase runway throughput through system stability, predictability and precision spacing. This paper describes problems and costs associated with an imprecise arrival flow. It also discusses methods by which Air Traffic Controllers achieve and maintain an optimum inter- arrival interval, and explores means by which AMSTAR can assist in this pursuit.

AMSTAR is an extension of NASA's previous work on in-trail spacing that was successfully demonstrated in a flight evaluation at Chicago O’Hare International Airport in September 2002. In addition to providing for precision inter-arrival spacing, AMSTAR provides speed guidance for aircraft on converging routes to safely and smoothly merge onto a common approach.

Much consideration has been given to working with operational conditions such as imperfect ADS-B data, wind prediction errors, changing winds, differing aircraft types and wake vortex separation requirements.

A series of Monte Carlo simulations are planned for the spring and summer of 2004 at NASA Langley to further study the system behavior and performance under more operationally extreme and varying conditions. This will coincide with a human-in-the-loop study to investigate the flight crew interface, workload and acceptability.

\section{INTRODUCTION}

Our air traffic system currently moves more than 700 million passengers annually [1]. Traffic levels at our nations busiest airports are escalating at a historic rate, with nine of our 35 major airports operating above 9-11-01 levels [2]. Additionally, seven of the top ten airports in the US are expected to return to pre-9-11 levels by the end of 2004. As increased security measures and cheaper airfares fuel this growth, there are a number if initiatives that are underway to support it. To accommodate this continuing demand on airport capacity, runways are being built and plans are in the works for many more. While the addition of runways is one way to increase capacity for an airport, work 
must begin many years in advance before the first aircraft can land on the new concrete. Challenges, such as the "not in my backyard" mentality, mean that additional runways face stiff opposition from local home and business owners' groups due to the increased noise that they bring. In addition to adding new runways, other complimentary alternatives are being actively pursued that involve new procedures that capitalize on the technical possibilities current technology and future advances may offer. One such venture is NASA's Distributed Air/Ground Traffic Management (DAG-TM) concept under the Advanced Air Transportation Technologies (AATT) Project. The DAG-TM concept involves various levels of collaboration between airborne and ground-based resources to enable less-restricted and more efficient aircraft trajectories throughout all phases of flight.

One aspect of DAG-TM focuses on terminal arrival operations, providing a means for merging multiple inbound streams and delivering precisely spaced aircraft to the runway threshold. Properly equipped aircraft and trained flight crews are able to use speed guidance cues, advanced displays, and lateral path changes to meet these goals. This concept also envisions advanced ground-based decision support tools. These ground-based tools and associated procedures are being developed at the NASA Ames Research Center.

\section{Problems Associated with an Imprecise Arrival Flow}

The ground tempo at major airports is a precisely timed and orchestrated movement of aircraft, baggage, cargo, fuel, and supplies. Aircraft have to be at specific locations, at precisely the right time, for these items to be loaded and unloaded, and for passengers to make connecting flights. At congested airports that are being forced to accept more and more flights each day, support personnel (fuelers, baggage handlers, caterers etc.) are tightly scheduled and have little time during heavy arrival and departure pushes to accommodate a late arriving flight. A flight that arrives at its gate late may have to wait for fuel trucks to arrive and baggage handlers to unload and reload. Even worse, the late arrival may still be parked at the gate when the next aircraft arrives at its scheduled time, causing further down-line disruptions.

In many instances, late arrivals at the gate can be attributed to arrival flow factors. An arrival flow that does not put aircraft over the threshold at the proper time, or has them landing on a runway other than originally intended can lead to late or early gate arrival times. Conversely, a predictable arrival flow, where aircraft are delivered to the intended landing runway at a specified time, enables ground controllers to issue the most efficient taxi routes, in turn reducing taxi time and costly fuel consumption. Working back through the arrival flow, it's important to consider what factors might affect a controller's ability to deliver aircraft over the threshold at the optimum rate and on the intended landing runway.

Controllers typically segregate aircraft into different arrival paths based on performance characteristics. In cases where runway configuration does not allow controllers to send dissimilar arrivals (i.e. turboprops vs. jets) to different runways, arrivals are merged at the latest point possible when performance between the types is more compatible.

\section{Arrival Path Options}

As a controller works aircraft through the terminal airspace, they have only a limited set of methods by which they can alter an aircraft's position within a flow or the spacing of an aircraft between its lead and follower. Those methods are further constrained by factors such as airspace configuration, aircraft type and performance, meteorological conditions, and the number and configuration of runways.

Depicted in Figure 1 is a generic airport. The solid lines show the normal arrival paths, and the dashed lines show those that a controller could use to shorten or lengthen an arrival's flight path, thus positioning it at just the right spot in an arrival flow. Those alternatives include extending flight legs, cutting corners, turns off path, multiple turns (Sturns), 360 degree turns, and diversions to alternate runways. Vertical separation is often used to assure separation requirements while the lateral means described above are employed. However, given all of these spacing techniques, the most common method for regulating inter-arrival spacing is speed. 
Speed commands are issued to either increase/decrease to a specified speed or by a specified increment. Frequently, combinations of path deviations, vertical separation, and speed commands are used to set and maintain proper inter-arrival spacing. But there are two issues associated with this technique.

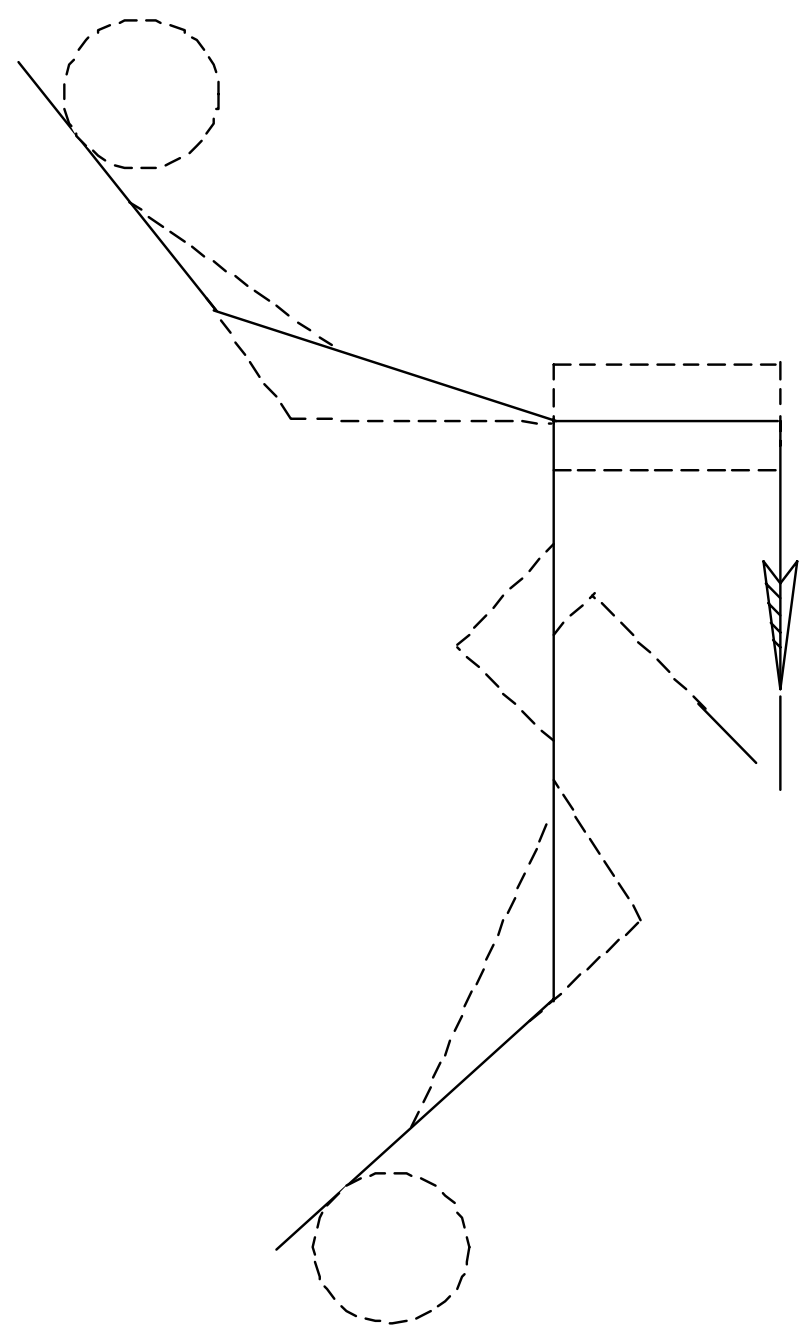

Figure 1. Pattern Legs and Options

First, when a controller considers using any of speed, lateral path, or vertical commands, factors such as wind and other meteorological conditions, aircraft performance limitations, and the location of other traffic must be taken into account to select the most appropriate method or combination of methods [3]. For example, in the case of a higher performance aircraft in descent, closing rapidly on a lower performance aircraft, a lateral path maneuver may be more appropriate than a speed reduction due to the faster aircraft's inability to descend and slow simultaneously. The controller must recognize this and select the proper technique. Once the maneuver is selected and transmitted to the aircraft, the execution and progress of the maneuver must be monitored for its desired effect. Almost all of the techniques discussed here also require a "normalizing" command. Aircraft that are vectored off path must be vectored again to re-intercept the nominal path. Speed commands must be normalized once the desired spacing is achieved. All of this requires the controller's close attention and accurate timing.

Secondly, most manual speed adjustments and maneuvers off of the nominal arrival path are less efficient than a smooth, planned arrival. Longer paths use more fuel. Deploying aircraft flaps, landing gear, or speed brakes to decelerate adds to noise and detracts from passenger comfort. Complying with multiple ATC commands for any of the above adds to flight crew workload.

\section{Closing the Gap}

When a terminal controller takes a hand-off from the Center controller, aircraft are typically spaced according to en-route minima, which is higher than that required in the terminal area. The terminal controller is then left with the task of "closing the gap" and compressing the arrival flow, primarily using speed commands. Normally handoffs are taken with the aircraft flying at higher airspeeds, and the controller then issues speed reductions at the appropriate time to assure proper spacing. The goal at that point is to get the aircraft as close as they can, as quickly as they can. But there are drawbacks to this approach. When controllers are trying to compress the arrival flow to account for lower terminal area separation minima, they have to closely monitor closure rates, particularly when those rates are higher than normal. Since controllers will not normally put aircraft in situations where they could potentially lose separation, the controller will slow the closing aircraft at a point that will leave it with a separation interval that is greater than the required minima; a separation buffer. The size of this buffer is highly dependant on the experience of the controller. This is a tedious operation that requires significant mental resources on the part of the controller, and at 
the same time leaves the aircraft at greater than the required minima. If the controller issues the speed reduction too soon, precious arrival route space goes unused and often remains unused.

Once the initial compression is accomplished and aircraft are established in the flow at more stable speeds, a controller might then attempt to refine the spacing by issuing smaller speed changes to tighten the flow. Again, the controller must monitor the closure rate and return the aircraft to normal speeds at the precise time. This method of speed control adds significantly to controller workload, effects fuel consumption and passenger comfort, and increases noise.

\section{Merging}

Merging arriving traffic flows can be one of the most challenging controller functions in the terminal area. The controller must pay constant attention to the arrival mix, the direction of heaviest flow including flow trends, the forecast and actual winds, while constantly coordinating with upstream controllers to achieve the appropriate flow rates.

Controllers have aircraft track and position predictors that are helpful in managing this operation, but these tools have their deficiencies. These decision support tools must be applied to each pair of merging aircraft. Since the predictors are extrapolated vectors based on radar returns, they are only updated with each sweep of the radar antenna, and they don't take into account planned changes in aircraft trajectory. If an aircraft is on a leg of an arrival that makes a turn immediately prior to the merge point, the tool's usefulness is limited.

\section{Precision, Time-Based Self- Spacing}

NASA has been studying concepts for selfspacing of aircraft operating in airport terminal areas since the 1970s. Interest in these concepts has recently been renewed due to a combination of the continued growth in air traffic and increasing demand on airport (and runway) throughput, the emergence of enabling technology (Automatic Dependent Surveillance Broadcast data link, ADSB), and the encouragement by the FAA's Safe Flight 21 Program to examine airborne approach spacing concepts. Currently, Concept Element 11 of DAG-TM is investigating airborne technologies and procedures that will assist the pilot in achieving precise inter-arrival spacing behind another aircraft. This new spacing clearance instructs the pilot to follow speed cues from a new on-board guidance system called Airborne Merging and Spacing for Terminal Arrivals (AMSTAR). AMSTAR receives Automatic Dependent Surveillance-Broadcast (ADS-B) reports from the leading aircraft and calculates the appropriate speed for the following aircraft to fly to achieve the desired time or distance-based spacing interval at the runway threshold.

An early part of this concept, begun at NASA Langley in 1999, was called Advanced Terminal Area Approach Spacing (ATAAS). It was based on the idea that a following aircraft, on the same flight path as the lead, would maintain a time-based, rather than distance-based, spacing interval from the preceding aircraft [4]. Evaluation of this concept was conducted in a high-fidelity, B757 full mission simulator using eight airline subject pilots [5]. The results of this simulation study showed that the ATAAS guidance provided a means for achieving a target threshold arrival interval within \pm 5 seconds (this equates to approximately $1100 \mathrm{ft}$ at the approach speed of $130 \mathrm{kt}$ ) across all test conditions. When autothrottles were used to track the ATAAS guidance, a mean error within \pm 1 sec, equivalent to $220 \mathrm{ft}$., was achieved. The standard deviation was 2 seconds. For comparison purposes, a simulator study conducted at Langley in 1990 using conventional air traffic control methods and ground-based automation resulted in a delivery precision of approximately 12 seconds [6]. A later evaluation of the ATAAS concept was conducted at the Chicago O'Hare International Airport and its surrounding terminal area [7] with results similar to the full motion simulator.

The ATAAS concept (and the follow-on AMSTAR development) was based on the premise that the ultimate operational goal was not to accurately and precisely space individual pairs of aircraft, but rather to achieve a system-wide improvement in performance. The overall benefit of this approach will be realized by obtaining better consistency in spacing from a system-wide standpoint, sometimes at the expense of having excessive spacing between individual aircraft pairs. 
As such, under normal circumstances, no single aircraft would be given guidance to aggressively achieve a spacing interval beyond what would normally be expected in current-day operations. It should be readily apparent that increasing the speed of one aircraft excessively in order to "close up the gap” with a preceding aircraft could quickly destabilize the system by multiplying the effect on the speed required of every aircraft that is in-trail, creating increasingly larger gaps and speeds well beyond acceptable operational standards. Of course there may occasionally be operational reasons why a controller may need an aircraft to more aggressively capture a specified interval than that commanded by the tool. That capability is envisioned for future versions of AMSTAR and will be discussed later.

With ATAAS, aircraft had to be on the same arrival path to take advantage of its capabilities. As a logical second generation of that technology, AMSTAR, as the name implies, adds the merging capability. Aircraft no longer need to be on the same path to apply time-based spacing. This new capability offers two benefits: (1) it would increase the time available for aircraft to achieve the desired spacing (notionally to the entire time they are within the terminal area) and (2) it could be used to ensure proper merging of arrival streams, potentially reducing the controllers task from active vectoring for the merge, to monitoring the progress of an airborne-managed merge.

\section{Arrival Route Procedures and Interval Assignment}

An initial assumption in this concept is that arriving aircraft would be metered to the terminal boundary using a ground-based metering tool. Aircraft would be time-spaced according to meteorological conditions, runway configuration and other factors. Aircraft would then fly a charted Standard Terminal Arrival Route (STAR), similar to those in use today. This arrival route would be extended to include a complete lateral path to the final approach course, plus a vertical profile (speed and altitude), all of which become part of the arrival clearance. Figure 2 depicts such an arrival.

The controller would then issue an additional clearance to AMSTAR-equipped aircraft, which identifies the traffic to follow and the assigned spacing interval. Theoretically, this clearance could be issued at any time during the arrival. The interval would be a value that the terminal facility assigns based on traffic volume, meteorological conditions, local needs (e.g., departures on same runway), wake turbulence minima, and other factors. It is envisioned that future wake turbulence detection and prediction technologies could be used in the calculation of the interval, assigning each aircraft a unique wake turbulence separation minima. It is also reasonable to assume that using advanced ground-side decision support tools, a different "optimum" interval, down to the second, could be issued to each aircraft, based on the needs of the air traffic situation.

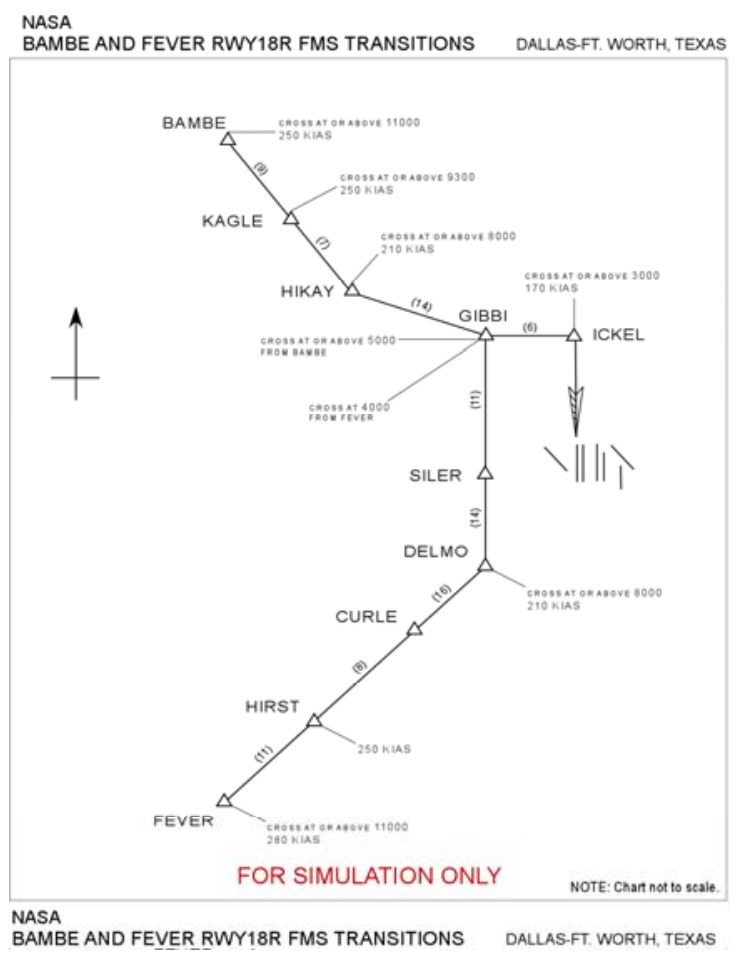

Figure 2. Typical Precision Spacing STAR

The term optimum is used because "optimum" doesn't necessarily mean "minimum”. Minimum would imply the lowest separation allowable, given the flight conditions, aircraft types, etc. Optimum, on the other hand, takes into account many other factors such operational requirements, runway occupancy times and the like. Under certain, and perhaps most conditions, the minimum may be the optimum. But the point is that ATC can now 
devote their effort toward "optimizing" the interval, and have a high degree of expectation that it will be met.

Precise inter-arrival spacing can have seemingly small, but meaningful arrival capacity gains. For example, if the throughput for a runway with a demand rate (ratio of arrivals to throughput) of $85 \%$ could be increased by only $5 \%$, the mean delay times for arriving aircraft could be reduced by as much as $29 \%$ [8]. The significance of this example is that if we could increase the runway throughput from 30 to 32 aircraft per hour, we could reduce the arrival delays by over $30 \%$ by just landing these 2 more aircraft.

Secondly, spacing intervals that are optimum for each aircraft pair may now be used. While this capacity increase would not be as significant as that gained by increasing the inter-arrival precision, there would still exist an advantage in using this capability.

Each aircraft participating in AMSTAR operations would need to transmit its state data (position and velocity), final approach speed, weight/wake-vortex class, wind data, and the name of its arrival route. This information would then be used by a following aircraft to compute a speed command for spacing. ADS-B is the broadcast means for this information. If the transmitting aircraft is using AMSTAR and spacing behind another aircraft, it would also transmit the ID of its lead aircraft, the assigned spacing, and information on the AMSTAR operational mode. Note that while ADS-B has been used in the development of this initial concept, non-ABS-B aircraft could participate as lead aircraft if their required data could be provided by another means, e.g., Traffic Information System - Broadcast (TIS-B).

Since traditional operational considerations dictate a stabilized speed prior to touchdown, AMSTAR also transitions into a "Final" mode once the aircraft has crossed the Final Approach Fix, providing speed guidance for a stabilized final approach segment.

\section{An Airborne Controller Tool}

In a sense, AMSTAR can be viewed as an airborne controller tool in that it performs many of the calculations that a controller currently does, with the exception that these calculations are made on the flight deck, at the direction of ATC, instead of in a centralized ground-based system. As discussed in the previous sections, a controller is constantly assessing an arrival flow, modifying airspeeds, altitudes and flight paths based on those assessments, and monitoring all aircraft for execution and performance of those modifications. This is a highly complex process because it is a multi-iteration process for each aircraft, and iterations for each aircraft rarely coincide with that of any other. A controller may be watching the closure rate of one aircraft on the one ahead of it, issuing a slowing command to another, and waiting for just the right moment to issue a vector onto base leg for another. Frequently, many instances of each of these and other tasks are occurring at once.

Proficiency as a terminal controller takes years of experience. The "calculations" necessary to create a safe, stable arrival flow become less like calculations, and more of a "sense. Controllers have to become intricately familiar with the aircraft that are common to the airport, traffic patterns and procedures needed to accommodate heavy flows, and meteorological conditions that are particular to that region. Techniques to work traffic may literally change with the seasons. And although AMSTAR does not replace a controller, it does help shift some of the complexity away from the job.

In a two-aircraft, in-trail scenario, AMSTAR makes the same calculations that that a controller would. It computes the error between the leading and following aircraft based on the difference between how far behind the lead the trailing aircraft is relative to how far it should be. It provides a commanded speed to the trailing aircraft to attain the assigned interval by the threshold and monitors it for success. While the AMSTAR speed commands will always provide safe separation, additional alerting is also provided for closure rates and distances that could jeopardize the separation minima. This alerting provides a second layer of protection from separation loss. Lastly, AMSTAR also provides a stable, predictable speed profile and behavior, around which other traffic can be worked.

As mentioned above, AMSTAR cannot replace a controller; nor was it designed to. It does not strategize, it does not plan beyond the aircraft it's tracking as its lead, and like any other equipment, it 
can break. But it does have the potential to be a valuable controller tool.

Other technologies have provided similar benefits to controllers. For instance, the Instrument Landing System (ILS) is a tool that controllers rely on to accurately deliver aircraft to the runway. Once the clearance is issued and aircraft are established on final, the controller needs only to monitor the aircraft on the approach, acting as a safeguard against system failures, and handling missed approaches if they occur. User Request Evaluation Tool (URET) and tools associated with the Center- TRACON Automation System (CTAS) are tools that perform functions and calculations that Center controllers once had to perform manually. Flight Management Systems (FMS) aboard aircraft give aircraft the capability to more accurately meet fix crossing times, altitudes, or airspeeds to meet air traffic control requirements.

\section{Flexibility}

There are several facets of the AMSTAR concept that give controllers the continued flexibility they need. As described earlier, in an effort to maintain overall system stability and to avoid large speed variances among aircraft on the same or merging paths, AMSTAR limits the speed guidance commanded to remain within 10 percent of the speed defined for that segment of the arrival. However, there are often instances when it may be operationally advantageous to a controller for an aircraft to more aggressively capture the assigned spacing interval. There are capabilities envisioned for later versions of the tool to allow an aircraft to fly at speeds higher than the $10 \%$ limit, and then slow at a pre-determined point, speed, or time interval. The question then arises that if one aircraft is directed to capture assigned spacing at a more aggressive rate, then what happens to those that may be spacing on that aircraft?

AMSTAR will actively space on another aircraft that has at least ADS-B out, on the same or a different arrival route, at any assigned interval. Therefore, a controller can amend the assigned interval of a trailing aircraft at any point in the pattern. Of course, the closer the aircraft is to the final approach fix, the less time it's going to have to successfully attain that interval by the time it reaches the threshold. As a result, large-scale fluctuations of groups of aircraft in the arrival stream can be avoided when using a more aggressive capture mode on a single aircraft by simply assigning a greater interval to the aircraft in trail.

One AMSTAR function that was originally a part of ATAAS, is the capability to actively space behind a lead aircraft that has been vectored off of the STAR. For instance, if three aircraft are in-trail, and numbers two and three are actively spacing off of the aircraft in front of them, a controller could vector the first aircraft off the arrival route. The second aircraft then tracks the first using "breadcrumbs", a navigation display depiction of the lead aircraft's lateral path, and follows the speed guidance to maintain the assigned interval. The third aircraft in the string would do the same thing reference the second, and so on. Thus, the controller needs only to vector the lead aircraft of an in-trail string, and the others, if so cleared, will follow. This functionality could be very useful when the controller is vectoring aircraft around weather or special operations airspace. This could also reduce the workload associated with runway changes or other delay causing events while enhancing the operational efficiency when these events occur.

Since AMSTAR is a flight deck tool, it might be incorrectly assumed that the tool will benefit only the controller and the AMSTAR equipped, self-spacing aircraft. In practice, potential benefits for non-equipped aircraft exist as well. Assume that aircraft 1 and 3 in a three aircraft stream are AMSTAR equipped, aircraft 2 is equipped for IFR flight only, and all aircraft are being spaced at 120 seconds (arbitrary number chosen for this example). Initially it may seem to make sense to move 3 behind 1 and then vector 2 using traditional techniques. Another method, though, would be to keep the original sequence and space 3 behind 1 at 240 seconds. This means that the slot for aircraft 2 is completely protected. At that point it's much easier to monitor 2's position in the flow, given that the interval between 1 and 3 will remain at a predictable 240 seconds.

\section{Planned Studies}

Two studies are underway at NASA Langley Research Center to characterize the performance 
and usability of the AMSTAR concept. A fast-time simulation (Monte Carlo) is examining the performance under varying conditions to identify situations where speed intervention is not sufficient for precision spacing. Under these extreme conditions the concept might need to be augmented to include limited maneuverability by the aircraft to meet the operational goals.

A human-in-the-loop study has recently been conducted to determine the flight crew and ATSP acceptability of the concept. Three airspace and arrival route structures were used for this work. The structures are modeled after those found at LaGuardia, Chicago-O’Hare, and San Francisco International airports. They are not intended to simulate traffic flows or procedures at those actual locations. Instead, they were selected because each offered flight paths and legs of varying lengths, merge points with different intercept trajectories, and different vertical profiles.

The human-in-the-loop study was conducted in the NASA Langley Air Traffic Operations Laboratory (ATOL). There were two evaluation sessions with each session having nine airline subject pilots flying approaches into the three airports, using medium fidelity desktop flight simulators. Each session included multiple approaches into each of the three airports. In addition to the pilots, for each session, two current or retired terminal area Air Traffic Controllers were used. Their tasks included issuing clearances to the subject pilots, and noting observed irregularities. They were also asked to provide opinions on controller/pilot interaction, potential enhancements from a controller perspective, and procedures that might further enable the concept. The results of this evaluation are currently being analyzed.

\section{Conclusion}

A terminal area ATC facility takes aircraft from the Air Route Traffic Control Center (ARTCC) and prepares the arrival flow for landing. The controllers compress the arrival flow by reducing the separation distance between aircraft from the center requirements to that of the terminal area. They apply a number of techniques to accomplish this, to include extending and shortening flight legs, and issuing speed commands. In the application of these techniques, they must consider such factors as wind and other meteorological conditions, aircraft types and performance characteristics, and the wake turbulence separation criteria. Proper judgment and timing are critical to the success of the operation. Excessive spacing between aircraft and improperly applied spacing techniques cost airlines valuable fuel, increase flight delays, and add to controller workload and frustration.

A new operational concept for terminal area arrivals is being proposed that would allow for increases in runway throughput by increasing the precision with which aircraft are spaced at the runway threshold. This precision spacing operation uses on-board speed guidance to obtain an assigned inter-arrival spacing. This prototype concept and supporting tool is called Airborne Merging and Spacing for Terminal Arrivals (AMSTAR), and it allows for spacing operations to commence before the aircraft are physically in-trail, i.e. allows the merging of different streams of traffic. These operations are enabled by the advent of advanced surveillance and datalink capabilities such as ADS-B. In addition to improved precision at the runway threshold, these operations could allow for more dynamic and optimized spacing for each pair of arriving aircraft. This optimal spacing interval could be a combination of wake turbulence avoidance and runway occupancy times. The capability to more precisely space aircraft at the runway threshold can allow for greater runway utilization, offering potentially large operational savings by reducing arrival delay times. In addition, precision spacing operations could result in fewer clearances being issued to equipped aircraft, thus decreasing radio traffic and the associated workload for both the controllers and the pilots.

\section{References}

[1] GAO Report to the Chairman and Ranking Democratic Member of the Subcommittee on Aviation, House Committee on Transportation and Infrastructure, June 2002. "Air Traffic Control, FAA Needs to Better Prepare for Impending Wave of Controller Attrition”.

[2] Capacity Needs in the National Airspace System Report, An Analysis of Airport and Metropolitan 
Area Demand and Operational Capacity in the Future, FAA, June 2004

[3] FAAO 7110.65, Air Traffic Control.

[4] Abbott, T. S. July 2002, Speed Control Law for Precision Terminal Area In-Trail Self Spacing, NASA TM 2002-211742,.

[5] Oseguera-Lohr, Rosa M., Lohr, Gary W., Abbott, Terence S., and Eischeid, Todd M.

Evaluation Of Operational Procedures For Using A Time-Based Airborne Interarrival Spacing Tool

AIAA-2002-5824.

[6] Credeur, L., J. A. Houck, W. R. Capron, and G. W. Lohr , 1990, Delivery Performance of Conventional Aircraft by Terminal Area, TimeBased Air Traffic Control, A Real-time Simulation Evaluation. NASA/TP-2978.

[7] Lohr, Gary W., Oseguera-Lohr, Rosa M., Abbott, Terence S., and Capron, William R. Flight Evaluation of a Time-Based Airborne Interarrival Spacing Tool. $5^{\text {th }}$ EUROCONTROL / FAA ATM R\&D Seminar, Budapest, Hungary, June 23-27, 2003.

[8] Credeur, L., 1977, Basic Analysis of Terminal Operation Benefits Resulting from Reduced Vortex Separation Minima. NASA/TM-78624. 